\title{
DECIDING REGULARITY OF THE SET OF INSTANCES OF A SET OF TERMS WITH REGULAR CONSTRAINTS IS EXPTIME-COMPLETE
}

\author{
OMER GIMÉNEZ*, GUILLEM GODOY*, AND SEBASTIAN MANETH ${ }^{\dagger}$
}

\begin{abstract}
Finite-state tree automata are a well studied formalism for representing term languages. This paper studies the problem of determining the regularity of the set of instances of a finite set of terms with variables, where each variable is restricted to instantiations of a regular set given by a tree automaton. The problem was recently proved decidable, but with an unknown complexity. Here, the exact complexity of the problem is determined by proving EXPTIME-completeness. The main contribution is a new, exponential time algorithm that performs various exponential transformations on the involved terms and tree automata, and decides regularity by analyzing formulas over inequality and height predicates.
\end{abstract}

Key words. EXPTIME complexity, regularity, terms with variables, pattern matching, regular constraints

AMS subject classifications. 68Q17, 68Q42, 68Q45

1. Introduction. Finite representations of infinite sets of terms are useful in many areas of computer science. The choice of formalism for this purpose depends on its expressiveness, but also on its computational properties. Finite-state tree automata (TA) 6, 2] are a well studied formalism for representing term languages, due to their good computational and expressiveness properties. They characterize the "regular term languages", a classical concept used, e.g., to describe the parse trees of a contextfree grammar or the well-formed terms over a sorted signature [12, to characterize the solutions of formulas in monadic second-order logic [4, and to naturally capture type formalisms for tree-structured XML data [13, 1]. Similar to the case of regular sets of words, regular term languages have numerous convenient properties such as closure under Boolean operations (intersection, union, negation), decidable properties such as finiteness and inclusion, and they are characterized by many different formalisms such as regular grammars, regular term expressions, congruence classes of finite index, deterministic bottom-up TA, nondeterministic top-down TA, or sentences of monadic second-order logic [2]. Deterministic TA, for instance, can be effectively minimized and give rise to efficient parsing.

When the used formalism for representing an infinite set of terms is not a TA, it is often expedient to decide whether the represented set is in fact regular. A simple and natural way of describing an infinite set of terms, is through the use of "patterns". A pattern is a term with variables; it describes all terms obtained by replacing the variables by (variable-free) terms; see, e.g., [11, 10, and the references given there. Term patterns are used for pattern matching in most modern programming languages, and were already present in very early languages such as LISP. They are a central concept in compiling, natural language processing, automated deduction, term rewriting, etc. In some of these applications, variables in patterns are restricted to be replaced by terms in a regular language. E.g. in a programming language with regular types

\footnotetext{
${ }^{*}$ Universitat Politècnica de Catalunya, Barcelona, Spain (\{ogimenez, ggodoy\}@lsi.upc.edu). The second author was supported by Spanish Min. of Educ. and Science by the FORMALISM project (TIN2007-66523) and by the LOGICTOOLS-2 project (TIN2007-68093-C02-01).

$\dagger$ NICTA and University of New South Wales, Sydney, Australia (sebastian.maneth@nicta.com.au)
} 
(see, for instance, 8, 9]), variable instances might be constrained to regular term languages. Typically, term patterns in a programming language must be linear (i.e., every variable occurs at most once) in order to guarantee that the resulting type is regular. Our result shows that even if non-linear patterns are allowed (which is the case in logic programming languages such as Prolog), one can statically determine regularity, i.e., the existence of an exact regular type, in exponential time.

More precisely, we consider the problem of determining the regularity of the set of instances of a set of terms with regular constraints, which we abbreviate as the "RITRC" problem. A particular case of this problem, in which variables can be replaced by arbitrary terms (without variables), was considered in [11] and shown to be coNP-complete (cf. also [10]). The general RITRC problem was recently proved decidable [7. The complexity of their decision procedure was left open in [7, but can easily be seen to exceed exponential time. Moreover, their solution is based on a rather general result of [3] about first-order formulas with regular constraints, for which the complexity is not known.

In this paper, we determine the complexity of the RITRC problem by proving that it is EXPTIME-complete. At the beginning of Section 3 we show that the RITRC problem is EXPTIME-hard. This is done via a straightforward reduction from the finite intersection emptiness problem for tree automata. The remaining part of Section 3 describes an EXPTIME algorithm solving the problem, starting with an overview of it in Section 3.1. In summary, the algorithm first changes the regular constraints from several TA to one single tree automaton (of exponential size) with special properties. It then picks a non-linear term $s$ from the given set $S$ of terms, and checks the "infinite instances property of $s$ in $S$ ": are there infinitely many instantiations of a non-linear variable $x$ in $s$, which are not instances of $S-\{s\}$ (under the regular constraints)? If the infinite instances property holds for some $s$ in $S$, then our algorithm stops and we know that the set of terms represented by $S$ (under the regular constraints) is not regular. Otherwise, we can replace $s$ by a new term $s^{\prime}$ that is linear in the variables, i.e., which does not contain duplicated variables. Roughly speaking, our algorithm then starts over again, with the new set $(S-\{s\}) \cup\left\{s^{\prime}\right\}$. In this way, the algorithm will construct a set $S^{\prime}$ of terms in which all terms are linear in the variables, if and only if the represented set is regular. To check the infinite instances property of $s$ in $S$, we instantiate the term $s$ at all nonvariable positions of terms in $S-\{s\}$, and then formulate inequality constraints of the resulting terms with terms of $S-\{s\}$. It is a non-trivial task to efficiently solve such inequality constraints. In fact, in order to solve systems of such inequality constraints in EXPTIME, it was a crucial step for us to introduce additional height constraints on the variables of the inequality constraints. The final formula $F$ over height and inequality predicates characterizes all instances of $s$ that are not instances of terms in $S-\{s\}$. Our algorithm solves the RITRC problem in exponential time by iteratively constructing and solving such formulas $F$.

2. Preliminaries. The size of a set $S$ is denoted by $|S|$. A signature consists of an alphabet $\Sigma$, i.e., a finite set of symbols, together with a mapping that assigns to each symbol in $\Sigma$ a natural number, its arity. We write $\Sigma^{(k)}$ to denote the subset of symbols in $\Sigma$ that are of arity $k$, and we write $f^{(k)}$ to denote that $f$ is a symbol of arity $k$. The set of all terms over $\Sigma$ is denoted $T_{\Sigma}$ and is inductively defined as the smallest set $T$ such that for every $f \in \Sigma^{(k)}, k \geq 0$, and $t_{1}, \ldots, t_{k} \in T$, the term $f\left(t_{1}, \ldots, t_{k}\right)$ is in $T$. For a term of the form $a()$ we simply write $a$. For instance, if $\Sigma=\left\{f^{(2)}, a^{(0)}\right\}$ then $T_{\Sigma}$ is the set of all terms that represent binary trees with internal nodes labeled 
$f$ and leaves labeled $a$. We fix the set $X=\left\{x_{1}, x_{2}, \ldots\right\}$ of variables, i.e., any set $V$ of variables is always assumed to be a subset of $X$. The set of terms over $\Sigma$ with variables in $X$, denoted $T_{\Sigma}(X)$, is the set of terms over $\Sigma \cup X$ where every symbol in $X$ has arity zero. By $\operatorname{Vars}(s)$ we denote the set of variables that occur in $s$. By $|t|$ we denote the size of $t$, defined recursively as $\left|f\left(t_{1}, \ldots, t_{k}\right)\right|=1+\left|t_{1}\right|+\ldots+\left|t_{k}\right|$ for each $f \in \Sigma^{(k)}, k \geq 0$ and $t_{1}, \ldots, t_{k} \in T_{\Sigma}$, and $|x|=1$ for each $x$ in $X$. By height $(t)$ we denote the height of $t$, defined recursively as height $\left(f\left(t_{1}, \ldots, t_{k}\right)\right)=1+\max \left(\right.$ height $\left(t_{1}\right), \ldots$, height $\left.\left(t_{k}\right)\right)$ for each $f \in \Sigma^{(k)}, k \geq 1$ and $t_{1}, \ldots, t_{k} \in T_{\Sigma}$, height $(a)=0$ for each $a \in \Sigma^{(0)}$, and height $(x)=0$ for each $x \in X$. Given a term $f\left(t_{1}, \ldots, t_{k}\right) \in T_{\Sigma}$, its set of positions $\operatorname{Pos}(t)$ equals $\{\varepsilon\} \cup_{1 \leq i \leq k}\left\{i . p \mid p \in \operatorname{Pos}\left(t_{i}\right)\right\}$. Here, $\varepsilon$ denotes the root node, and $p . i$ denotes the $i$ th child of position $p$. The subterm of $t$ at position $p$ is denoted by $t / p$, and the symbol of $t$ at position $p$ is denoted by $t[p]$; we say that $p$ is labeled by $t[p]$. For instance, for $s=g(f(a, b), c), s / 1$ equals $f(a, b)$ and position 1.2 is labeled by $b$. For a set $\Gamma$, we use $\operatorname{Pos}_{\Gamma}(t)$ to denote the set of positions of $t$ that are labeled by symbols in $\Gamma$. In particular, we define for $t \in T_{\Sigma}(X)$ the sets $\operatorname{Pos}_{\mathrm{v}}(t)$ and $\operatorname{Pos}_{\mathrm{nv}}(t)$ of variable positions and non-variables positions as $\operatorname{Pos}_{X}(t)$ and $\operatorname{Pos}(t)-\operatorname{Pos}_{X}(t)$, respectively. E.g., for $s$ as above, $\operatorname{Pos}_{\{c\}}(s)=\{2\}$ and $\operatorname{Pos}_{\mathrm{v}}(s)=\emptyset$. When a position $p$ is of the form $p_{1} \cdot p_{2}$, we say that $p_{1}$ is a prefix of $p$. For a set of positions $P$, we denote by Prefixes $(P)$ the set $\left\{p \mid \exists p^{\prime}: p \cdot p^{\prime} \in P\right\}$. For terms $s, t$ and $p \in \operatorname{Pos}(s)$, we denote by $s[p \leftarrow t]$ the result of replacing the subterm at position $p$ in $s$ by the term $t$. For instance, $f(f(a, a), a)[1 \leftarrow a]=f(a, a)$.

A (deterministic) tree automaton (over $\Sigma$ ), DTA for short, is a tuple $A=$ $\langle Q, F, \Sigma, \delta\rangle$ where $Q$ is a finite set of states, $F \subseteq Q$ is the set of accepting states, $\Sigma$ is a signature, and $\delta$ is a set of transitions of the form $f\left(q_{1}, \ldots, q_{k}\right) \rightarrow q$, where $f \in \Sigma^{(k)}, k \geq 0$, and $q, q_{1}, \ldots, q_{k} \in Q$. Moreover, for each $f \in \Sigma$ and each $q_{1}, \ldots, q_{k} \in Q$ there exists at most one (and at least one if the automaton is complete) $q$ such that $f\left(q_{1}, \ldots, q_{k}\right) \rightarrow q$ is in $\delta$. The language $\mathrm{L}(A)$ recognized by $A$ is the set $\left\{t \in T_{\Sigma} \mid A(t) \in F\right\}$ where $A(t)$ is recursively defined as $A\left(f\left(t_{1}, \ldots, t_{k}\right)\right)=q$ if $f \in \Sigma^{(k)}, k \geq 0, t_{1}, \ldots, t_{k} \in T_{\Sigma}, f\left(q_{1}, \ldots, q_{k}\right) \rightarrow q$ is a transition in $\delta$, and, for each $i \in\{1, \ldots, k\}, q_{i}=A\left(t_{i}\right)$. Note that, when $A$ is not complete, $A(t)$ might be undefined. We also define, for $q \in Q$, the set $\mathrm{L}(A, q)=\left\{t \in T_{\Sigma} \mid A(t)=q\right\}$ of terms for which $A$ arrives to state $q$. Note that $\mathrm{L}(A, q) \cap \mathrm{L}\left(A, q^{\prime}\right)=\emptyset$ for all $q \neq q^{\prime}$. We also extend $A(t)$ to terms $t$ in $T_{\Sigma \cup Q}$ by assuming that the states $q \in Q$ have arity 0 and $A(q)=q$ for each $q \in Q$. A set of terms $L \subseteq T_{\Sigma}$ is regular if there exists a DTA $A$ such that $L=\mathrm{L}(A)$. The size $|\tau|$ of a transition $\tau=\left(f\left(q_{1}, \ldots, q_{k}\right) \rightarrow q\right)$ is $k+2$ and the size $|A|$ of $A$ is $|Q|+\sum_{\tau \in \delta}|\tau|$.

Given a DTA, it is decidable whether its recognized language is (i) empty, (ii) finite, or (iii) has cardinality $k$, for a given $k$. The corresponding constructions all run in polynomial time and are straightforward generalizations of the ones for classical finite (word) automata; proofs can be found in Theorems 1.7.4, 1.7.6, and 1.7.10 of [2]. The following computational problems, together with the running times, are a consequence of the same proofs.

Lemma 2.1. Let $A=\langle Q, F, \Sigma, \delta\rangle$ be a DTA and $k$ a natural number. Each of the following sets can be computed in polynomial time: non-emptyStates $(A):=\{q \in$ $Q \mid \mathrm{L}(A, q) \neq \emptyset\}$ in $\mathcal{O}(|Q|+|\delta|)$, infiniteStates $(A):=\{q \in Q|| \mathrm{L}(A, q) \mid=\infty\}$ in $\mathcal{O}(|Q| \cdot|\delta|)$, and countUpto $(A, k):=\{(q, \min (|\mathrm{L}(A, q)|, k)) \mid q \in Q\}$ in $\mathcal{O}(|Q| \cdot|\delta|)$.

Sets of Terms with Regular Constraints Let $V \subseteq X$ be a finite set of variables and $\Sigma$ a signature. A regular constraint (over $V$ and $\Sigma$ ) is a mapping $M$ that associates to every $x \in V$ a DTA over $\Sigma$. A solution of $M$ is a mapping 
$\varphi: V \rightarrow T_{\Sigma}$ such that, for each $x \in V, \varphi(x) \in \mathrm{L}(M(x))$. A set of terms with regular constraints (over $V$ and $\Sigma$ ) is a pair $\langle S, M\rangle$ where $S$ is a finite subset of $T_{\Sigma}(V)$ and $M$ is a regular constraint over $V$ and $\Sigma$. The language $\mathrm{L}(\langle S, M\rangle)$ of $\langle S, M\rangle$ is defined as $\{t \mid \exists \varphi, s:(t=\varphi(s) \wedge s \in S \wedge \varphi$ is a solution of $M)\}$. A term in $\mathrm{L}(\langle S, M\rangle)$ is also called an instance of $\langle S, M\rangle$.

The following result is due to [11], cf. also [10.

Proposition 2.2. Let $V \subseteq X, S$ a finite subset of $T_{\Sigma}(V)$, and $M$ the regular constraint that maps every $x \in V$ to the trivial DTA that recognizes $T_{\Sigma}$. Regularity of $\mathrm{L}(\langle S, M\rangle)$ is coNP-complete.

When analyzing complexity, with $\|S\|$ we refer to the sum of sizes of all terms in $S$, and with $\|M\|$ we refer to the sum of sizes of all DTA in the image of $M$. With $|S|$ and $|M|$ we refer, as usual, to the number of elements in the sets $S$ and $M$ (i.e. number of pairs of the set defining the mapping $M$ ). We also do the following assumption in order to ease the complexity analysis.

Assumption: The maximum arity of a function symbol in $\Sigma$ is 2. It is well known that any arbitrary tree can be coded as a binary tree of essentially the same size. Usual such codings (such as the one taking first-child to left-child and nextsibling to right-child) preserve regularity of sets of terms (see, e.g., Section 8.3.1 in 2]); moreover, it can be seen easily that the transformation of the regular constraints into this new binary signature produces an at most quadratic size increase.

3. Regularity of the instances of a set of terms with regular constraints. Let $\langle S, M\rangle$ be a set of terms with regular constraints. The "regularity of the instances of a set of terms with regular constraints problem", RITRC for short, asks whether or not the set $\mathrm{L}(\langle S, M\rangle)$ is regular. We know, by Proposition 2.2, that RITRC is coNPcomplete in the particular case that $M$ maps each variable to a DTA that accepts all terms. In general, i.e., with regular constraints, decidability of RITRC was proved in [7; however, the complexity remained open. The algorithm of [7] does not run in exponential time, and in fact it has a far worse complexity. In this section we show that RITRC is EXPTIME-complete. We start with the easy part by showing that RITRC is EXPTIME-hard.

THEOREM 3.1. RITRC is EXPTIME-hard.

Proof. Let $\Sigma$ be a signature with $\Sigma^{(2)} \neq \emptyset$ and let $A_{1}, \ldots, A_{n}$ be DTAs over $\Sigma$. It is well known that testing whether $\mathrm{L}\left(A_{1}\right) \cap \cdots \cap \mathrm{L}\left(A_{n}\right)=\emptyset$ is EXPTIME-complete, cf. Theorem 1.7.5 of [2]. It follows that "universality of union", i.e., testing whether $\mathrm{L}\left(A_{1}\right) \cup \cdots \cup \mathrm{L}\left(A_{n}\right)=T_{\Sigma}$ is EXPTIME-complete. This is because a DTA can easily be complemented in polynomial time (first complete the DTA by adding, for any missing transition, a transition to a new "sink" state; second, change $F$ into $Q-F$ ). We now reduce universality of union to RITRC. Let $A$ be any fixed DTA that recognizes $T_{\Sigma}$ and let $f \in \Sigma^{(2)}$. The set of terms with regular constraints $\langle S, M\rangle$, where

$$
\begin{gathered}
S=\left\{f(f(x, x), y), f\left(x_{1}^{\prime}, x_{1}\right), \ldots, f\left(x_{n}^{\prime}, x_{n}\right)\right\} \\
M=\left\{x_{1} \mapsto A_{1}, \ldots, x_{n} \mapsto A_{n}, x \mapsto A, y \mapsto A,\right. \\
\left.x_{1}^{\prime} \mapsto A, \ldots, x_{n}^{\prime} \mapsto A\right\},
\end{gathered}
$$

is regular if and only if $\cup_{1 \leq i \leq n} \mathrm{~L}\left(A_{i}\right)=T_{\Sigma}$. To see this, consider first the case where $\cup_{1 \leq i \leq n} \mathrm{~L}\left(A_{i}\right)=T_{\Sigma}$. Then $\mathrm{L}(\langle S, M\rangle)=\mathrm{L}\left(\left\langle\left\{f\left(x_{1}^{\prime}, x_{1}\right), \ldots, f\left(x_{n}^{\prime}, x_{n}\right)\right\}, M\right\rangle\right)=$ $\left\{f(s, t) \mid s, t \in T_{\Sigma}\right\}$, which is regular. In the other case, let $t$ be in $T_{\Sigma}-\cup_{1 \leq i \leq n} \mathrm{~L}\left(A_{i}\right)$. Intersect $\mathrm{L}(\langle S, M\rangle)$ with the regular set $\left\{f(s, t) \mid s \in T_{\Sigma}\right\}$. Since regular term languages are closed under intersection, the resulting set would be regular, if $\mathrm{L}(\langle S, M\rangle)$ 
was; but, the resulting intersection is $\left\{f\left(f\left(t^{\prime}, t^{\prime}\right), t\right) \mid t^{\prime} \in T_{\Sigma}\right\}$. By standard pumping arguments (see, e.g., Example 1.2.1 of [2]) this set is not regular. Thus, $\mathrm{L}(\langle S, M\rangle)$ is not regular in this case.

Proving that RITRC is in EXPTIME is considerably more complicated.

3.1. Overview of our algorithm for RITRC. Algorithm in [7]. In 7] decidability of RITRC was proved. We first explain the idea of that proof, and why it does not give rise to an EXPTIME algorithm. Then we give an overview of the algorithm presented in this paper. The following is the basic property used for deciding RITRC in [7] (and here).

DeFINITION 3.2. Let $\langle S, M\rangle$ be a set of terms with regular constraints. The term $s \in S$ satisfies the infinite-instances property in $\langle S, M\rangle$ if some variable $x$ has multiple occurrences in $s$, and there exists infinitely many instances $\varphi_{1}(s), \varphi_{2}(s), \ldots$ of $\langle\{s\}, M\rangle$ which are not instances of $\langle S-\{s\}, M\rangle$ and all of them different on $x$, i.e., $\varphi_{i}(x) \neq \varphi_{j}(x)$ for all $i \neq j$.

In [7] it was shown that the infinite-instances property is decidable and that it implies non-regularity of $\langle S, M\rangle$. To decide RITRC, the algorithm of [7] first looks for a term in $S$ with multiple occurrences of some variable $x$ satisfying $|\mathrm{L}(M(x))|=\infty$. If no such term exists, then it stops concluding regularity of $\mathrm{L}(\langle S, M\rangle)$ (note that in this case $\mathrm{L}(\langle\{s\}, M\rangle)$ is regular for each term $s$ in $S$, and regular sets are closed under union). Otherwise, it checks the infinite-instances property of $s$ in $\langle S, M\rangle$. In the affirmative case, it stops concluding non-regularity of $\mathrm{L}(\langle S, M\rangle)$. In the negative case, there are only a finite number of possible instantiations $\varphi(x)$ of each duplicated variable $x$ in $s$ providing a term in $\mathrm{L}(\langle\{s\}, M\rangle)$ and not in $\mathrm{L}(\langle S-\{s\}, M\rangle)$. Thus, by replacing $s$ by a finite number $\left\{s_{1}, \ldots, s_{k}\right\}$ of instantiations of $s$, the represented language $\mathrm{L}(\langle S, M\rangle)$ is preserved, and we obtain less duplicated variables. The algorithm in [7] decides regularity of $\mathrm{L}(\langle S, M\rangle)$ by iterating this process.

Estimating the complexity. To determine the complexity of the previous algorithm, we need to know how large is the number $k$ of instantiations of $s$, how large the terms $s_{1}, \ldots, s_{k}$ are, and, of course, how expensive it is to decide the infinite instances property. In [7, the latter is solved through a result of [3] about first-order formulas with regular constraints. The precise complexity of this result of [3] is not known, but it is expected to be higher than that of solving the infinite-instances property, since it solves a more general problem. We therefore devise our own algorithm for checking this property. But, also the sum of sizes of the terms $s_{1}, \ldots, s_{k}$ poses a problem, as it can grow iterated exponential, so the algorithm in $[7$ is certainly not in EXPTIME. One of the ideas of our new algorithm is hence not to replace $s$ by $\left\{s_{1}, \ldots, s_{k}\right\}$. Instead, we are able to find a "small" number $h$ (which depends on $S$ and $M$ ) such that all terms $s_{i}$ are guaranteed to be of height smaller than $h$. To take advantage of this fact, we add a new kind of constraint to $\langle S, M\rangle$ which allows duplicated variables $x$ of $s$ to be replaced only by "small" terms. The algorithm then continues on with this new system (called restricted regular constraints, see Definition [3.7), which has regular constraints plus height constraints on the variables.

Infinite-instances algorithm. How do we check the infinite-instances property of $s$ in $\langle S, M\rangle$ ? In Sections 3.4 3.5 and 3.6 we give an algorithm that solves this problem under several assumptions. To begin with, we require that the term $s$ is determined (see Definition 3.11 for the precise notion) in all the non-variable positions of terms in $S$. We also assume that the regular constraint $R$ is given by a single DTA $A$ (instead of the multiple ones in the image of $M$ ), and a mapping that associates variables with states of $A$. Finally, we require this DTA $A$ to satisfy the 1-or- $|S|$ prop- 
erty of Definition 3.5, which says that for any state $q$ of $A$, the cardinality of $\mathrm{L}(A, q)$ is either 1 , or it is greater than or equal to $|S|$. The reason for these assumptions is as follows. In order to decide the infinite-instances property, we compute a formula $\mathcal{F}$ whose solutions are the instances in $\mathrm{L}(\langle\{s\}, R\rangle)$ that are not in $\mathrm{L}(\langle S-\{s\}, R\rangle)$. This formula is a disjunction of conjunctions of inequalities, where each conjunction has at most $|S|-1$ inequalities. After some transformations on $\mathcal{F}$ by means of a system of inference rules, the variables $x$ with an associated state $q_{x}$ of $A$ satisfying $\left|\mathrm{L}\left(A, q_{x}\right)\right|=1$ disappear. Thanks to the 1-or- $|S|$ property, the remaining variables in $\mathcal{F}$ have at least $|S|$ possible instantiations. This fact is used to show that, for any surviving conjunction in $\mathcal{F}$, there is a variable instantiation that makes true the at most $|S|-1$ inequalities it is composed of, and variables with infinite language have infinite choices. Hence, we obtain that $s$ satisfies the infinite-instances property in $\langle S, R\rangle$ if the transformed formula $\mathcal{F}$ is not empty.

Overview of the algorithm. We give an outline of the EXPTIME algorithm that solves RITRC for a given instance $\left\langle S_{1}, M_{1}\right\rangle$. First of all, we transform $\left\langle S_{1}, M_{1}\right\rangle$ into $\left\langle S_{2}, R_{1}\right\rangle$ by preserving the represented language, where $R_{1}$ is a single regular constraint (Definition 3.3), and $S_{2}$ is the adaptation of $S_{1}$ from $M_{1}$ to $R_{1}$. Intuitively, $\left\langle S_{2}, R_{1}\right\rangle$ is the same problem stated with a single 1-or- $\left|S_{1}\right|$ DTA; the sizes of both $S_{2}$ and $R_{1}$ can be exponential with respect to the sizes of $S_{1}$ and $M_{1}$. This transformation is described in Section 3.2. The single regular constraint $R_{1}$ is then converted to a restricted regular constraint $R_{2}$, the new type of constraint, which we introduce in Section 3.3 that takes account of height restrictions.

The algorithm then proceeds as follows. At each step it picks a term $s$ of $S_{2}$ without height constraints, and with multiple occurrences of some variable $x$ satisfying $|\mathrm{L}(A, C(x))|=\infty$. If no term of this kind exists, then it stops concluding regularity of $\mathrm{L}\left(\left\langle S_{2}, R_{2}\right\rangle\right)$. Otherwise, it chooses a term $s$ satisfying the above conditions, and checks the infinite-instances property of $s$ with respect to $\left\langle S_{2}, R_{2}\right\rangle$. To do so, the algorithm loops over all possible partial instantiations $s_{i}$ of $s$ in the non-variable positions of $S_{2}$, and for each $s_{i}$, it finds a subset $S_{3} \subseteq S_{2}$, with $\left|S_{3}\right|=\left|S_{1}\right|-1$, such that $s_{i}$ has the infinite-instances property for $S_{2}$ if and only if it has the property for $S_{3}$. The fact that $\left|S_{3}\right|$ is small allows to check the infinite-instances property in exponential time. In the affirmative case the algorithm stops concluding non-regularity of $\mathrm{L}\left(\left\langle S_{2}, R_{2}\right\rangle\right)$. If no determination $s_{i}$ satisfies the infinite-instances property, the restricted regular constraint $R_{2}$ is modified so as to impose height constraints on the variables of $s$ with multiple occurrences. Since the number of terms with duplicated variables and without height constraints decreases, the iteration of this process decides regularity of $\mathrm{L}\left(\left\langle S_{1}, M_{1}\right\rangle\right)$. A careful analysis of all the steps involved will show that the time complexity is exponential.

3.2. Simplification to a single DTA. Recall from the preliminaries that we assume $\Sigma$ to be a fixed but arbitrary signature containing no symbol of arity greater than 2. We start with a set of terms with regular constraints $\left\langle S_{1}, M_{1}\right\rangle$ over a finite set of variables $V$. Recall that $S \subseteq T_{\Sigma}(V)$ is a finite set of terms and $M$ is a function that maps each $x \in V$ to a DTA over $\Sigma$. We now adapt this definition to a setting with only one single DTA $A$, and where variables in $V$ are now mapped to states in $A$. Moreover, we do not need accepting states anymore and simply drop them from $A$ 's definition (a "DTA without accepting states").

DeFinition 3.3. $A$ single regular constraint (over $V$ and $\Sigma$ ) is a pair $R=\langle A, C\rangle$, where $A=\langle Q, \Sigma, \delta\rangle$ is a complete DTA without accepting states and $C$ is a mapping $C: V \rightarrow Q$. The size $\|R\|$ of $R$ is $|V|+\|A\|$. A solution of $R$ is a mapping $\varphi: V \rightarrow T_{\Sigma}$ 
such that, for each $x \in V$, it holds that $\varphi(x) \in \mathrm{L}(A, C(x))$. A set of terms with single regular constraints (over $V$ and $\Sigma$ ) is a pair $\langle S, R\rangle$, where $S$ is a finite subset of $T_{\Sigma}(V)$ and $R=\langle A, C\rangle$ is a single regular constraint over $V$ and $\Sigma$. The language $\mathrm{L}(\langle S, R\rangle)$ of $\langle S, R\rangle$ is defined as $\{t \mid \exists \varphi, s:(t=\varphi(s) \wedge s \in S \wedge \varphi$ is a solution of $R)\}$. A term in $\mathrm{L}(\langle S, R\rangle)$ is also called an instance of $\langle S, R\rangle$.

Transforming a set of terms with regular constraints $\left\langle S_{1}, M_{1}=\left\{x_{1} \mapsto\right.\right.$ $\left.\left.A_{1}, \ldots, x_{n} \mapsto A_{n}\right\}\right\rangle$ into a set of terms with single regular constraints $\left\langle S_{2}, R_{1}\right\rangle$ satisfying $\mathrm{L}\left(\left\langle S_{2}, R_{1}\right\rangle\right)=\mathrm{L}\left(\left\langle S_{1}, M_{1}\right\rangle\right)$ is rather easy by considering the product automaton $A=A_{1} \times \cdots \times A_{n}$. But the size of $\left\langle S_{2}, R_{1}\right\rangle$ can be exponential in the size of $\left\langle S_{1}, M_{1}\right\rangle$. Moreover, it follows from Proposition 2.2 that regularity of $\mathrm{L}\left(\left\langle S_{2}, R_{1}\right\rangle\right)$ is at least NPhard. Hence, it is not enough to have an EXPSPACE-reduction from one problem to the other if we want to obtain an EXPTIME algorithm for the initial problem.

Thus, in the translation from $\left\langle S_{1}, M_{1}\right\rangle$ into $\left\langle S_{2}, R_{1}\right\rangle$ we keep in mind some additional properties obtained by the transformation process. For instance, the terms in $S_{2}$ are very similar to those in $S_{1}$ because they are obtained through variable renamings; we call this "structural similarity". Moreover, as mentioned in the outline of Section 3.1, we want the DTA $A$ to have the "1-or- $n$ " property, with $n=\left|S_{1}\right|$. We proceed to define both properties.

Definition 3.4. Let $V, V^{\prime}$ be sets of variables. A total function $\rho: V \rightarrow V^{\prime}$ is a variable renaming if it is injective, i.e., $\rho(x) \neq \rho(y)$ for $x \neq y$. For a term $s, \rho(s)$ is the term obtained from $s$ by replacing in s each variable $x \in V$ by $\rho(x)$. Two terms $s$ and $t$ are structurally similar, denoted by $s=_{\Sigma} t$, if $t=\rho(s)$ for a variable renaming $\rho$. For a set of terms $S$, StructDiff $(S)$ is the maximum number of non-structurally similar terms in $S$, i.e., $\operatorname{StructDiff}(S)=\max _{S^{\prime} \subseteq S \wedge\left(s, t \in S^{\prime} \Rightarrow s \neq \Sigma\right.}\left|S^{\prime}\right|$. Given a single regular constraint $R=\langle A, C\rangle$ we say that two terms $s$ and $t$ are structurally equal (with respect to $R$ ) if they are structurally similar, and $C(s[p])=C(t[p])$ for all $p \in \operatorname{Pos}_{\mathrm{v}}(s)$.

Note that if $s$ and $t$ are structurally equal with respect to $R$, then $\mathrm{L}(\langle\{s\}, R\rangle)=$ $\mathrm{L}(\langle\{t\}, R\rangle)$; the converse does not necessarily hold.

DeFinition 3.5. Let $A=\langle Q, \Sigma, \delta\rangle$ be a DTA. Let $n$ be a natural number. We say that $A$ is a 1-or- $n$ DTA if each state $q$ in $Q$ satisfies either $|\mathrm{L}(A, q)|=1$ or $|\mathrm{L}(A, q)| \geq n$.

Lemma 3.6. Let $\langle S, M\rangle$ be a set of terms with regular constraints. Then, $\langle S, M\rangle$ can be transformed in exponential time into a set of terms with single regular constraints $\left\langle S^{\prime}, R\right\rangle$ such that $\mathrm{L}\left(\left\langle S^{\prime}, R\right\rangle\right)=\mathrm{L}(\langle S, M\rangle)$ and the following properties hold.

- $R=\langle A, C\rangle$ satisfies that $A$ is a 1-or- $|S| D T A$.

- $A=\langle Q, \Sigma, \delta\rangle$ is complete and satisfies that $|Q| \leq\|M\|^{|M|} \cdot|S|$ and $|\delta| \leq$ $|\Sigma| \cdot|Q|^{2}=|\Sigma| \cdot\|M\|^{2|M|} \cdot|S|^{2}$

- $\left|S^{\prime}\right| \leq|S| \cdot|Q|^{|M|} \leq\|M\|^{|M|^{2}} \cdot|S|^{|M|+1}$

- Each term in $S^{\prime}$ is structurally similar to some term in $S$. In particular, StructDiff $\left(S^{\prime}\right) \leq|S|$.

- Every two distinct terms $s, t \in S^{\prime}$ are not structurally equal with respect to $R$.

- Each two distinct terms $s, t \in S^{\prime}$ do not share variables.

Proof. Let $M=\left\{x_{1} \mapsto A_{1}, \ldots, x_{n} \mapsto A_{n}\right\}$ and $A_{i}=\left\langle Q_{i}, F_{i}, \Sigma, \delta_{i}\right\rangle$ for $1 \leq i \leq n$. We first complete each DTA $A_{i}$ to a new DTA $A_{i}^{\prime}=\left\langle Q_{i}^{\prime}, F_{i}, \Sigma, \delta_{i}^{\prime}\right\rangle$ by adding a sink state and all undefined transitions to it. Recall the assumption that the maximum arity of $\Sigma$ is 2. Thus, $\left|Q_{i}^{\prime}\right|=\left|Q_{i}\right|+1$ and $\left|\delta_{i}^{\prime}\right|=|\Sigma| \cdot\left|Q_{i}^{\prime}\right|^{2}=|\Sigma| \cdot\left(\left|Q_{i}\right|+1\right)^{2}$. We now construct the product automaton (without accepting states) $A^{\prime}=\left\langle Q^{\prime}, \Sigma, \delta^{\prime}\right\rangle$, i.e., we set $Q^{\prime}=Q_{1}^{\prime} \times \cdots \times Q_{n}^{\prime}$ and if, for each $1 \leq i \leq n, \delta_{i}^{\prime}$ has the transition $f\left(q_{i, 1}, \ldots, q_{i, k}\right) \rightarrow$ 
$q_{i}$, then we add the transition $f\left(\left\langle q_{1,1}, \ldots, q_{n, 1}\right\rangle, \ldots,\left\langle q_{1, k}, \ldots, q_{n, k}\right\rangle\right) \rightarrow\left\langle q_{1}, \ldots, q_{n}\right\rangle$ to $\delta^{\prime}$. Since each state of $A^{\prime}$ is a tuple of $|M|$ states of the automata in $M$ plus a sink state, $\left|Q^{\prime}\right| \leq\|M\|^{|M|}$.

We then transform $A^{\prime}$ into a 1-or- $|S|$ DTA. To this end, we compute the mapping $M^{\prime}: Q \rightarrow\{1, \ldots,|S|\}$ with $M^{\prime}=\operatorname{count} \operatorname{Upto}\left(A^{\prime},|S|\right)=\{(q, \min (|\mathrm{L}(A, q)|,|S|)) \mid q \in$ $\left.Q^{\prime}\right\}$, according to Lemma 2.1. Now, using $M^{\prime}$ we obtain the desired $A$ as output of the following algorithm.

Input: $A^{\prime}=\left\langle Q^{\prime}, \Sigma, \delta^{\prime}\right\rangle$ and $M^{\prime}: Q^{\prime} \rightarrow\{1, \ldots,|S|\}$.

$Q:=\left\{q\left|q \in Q^{\prime} \wedge M^{\prime}(q)=\right| S \mid\right\} \cup$

$\left\{q^{i}\left|q \in Q^{\prime} \wedge 1 \leq i \leq M^{\prime}(q)<\right| S \mid\right\}$.

$\delta:=\emptyset$.

For each $q$ in $Q^{\prime}$ do:

If $M^{\prime}(q)=|S|$ then:

For each $f\left(q_{1}, \ldots, q_{m}\right) \rightarrow q$ in $\delta^{\prime}$ do:

For each $i_{1}, \ldots, i_{m}$ with $q_{1}^{i_{1}} \ldots, q_{m}^{i_{m}} \in Q$ do:

else: Add $f\left(q_{1}^{i_{1}}, \ldots, q_{m}^{i_{m}}\right) \rightarrow q^{1}$ to $\delta$.

Let $l_{1} \rightarrow q, \ldots, l_{k} \rightarrow q$ be all transitions of $\delta^{\prime}$ with $q$ as right-hand side.

counter: $=1$.

For each $i$ in $\{1, \ldots, k\}$ do:

Let $f\left(q_{1}, \ldots, q_{m}\right) \rightarrow q$ be $l_{i} \rightarrow q$.

For each $i_{1}, \ldots, i_{m}$ with $q_{1}^{i_{1}}, \ldots, q_{m}^{i_{m}} \in Q$ do:

Add $f\left(q_{1}^{i_{1}}, \ldots, q_{m}^{i_{m}}\right) \rightarrow q^{\text {counter }}$ to $\delta$.

counter++.

Complete $A=\langle Q, \Sigma, \delta\rangle$ and return the result.

It is clear that this algorithm generates a complete 1-or- $|S|$ DTA $A$ with $|Q| \leq$ $\|M\|^{|M|} \cdot|S|$, because at most $|S|$ new states are created for every state in $Q^{\prime}$. Moreover, since the maximum arity of $\Sigma$ is 2 , then at most $|\delta|=|\Sigma| \cdot|Q|^{2}$ transitions are possible with such number of states. The construction runs in exponential time because $A^{\prime}$ is constructed in exponential time, $M^{\prime}$ is constructed in time polynomial in $\left|A^{\prime}\right|$ by Lemma 2.1 and $A$ is constructed in time $\mathcal{O}(|A|)$.

Now, the set $S^{\prime}$ is obtained in the following way. Recall that the states $q$ in $Q$ are in fact of the form $q=\left\langle q_{1}, \ldots, q_{n}\right\rangle^{j}$, i.e., are tuples of states $q_{1} \in Q_{1}^{\prime}, \ldots, q_{n} \in Q_{n}^{\prime}$ plus an index $j$ satisfying $1 \leq j \leq M^{\prime}\left(\left\langle q_{1}, \ldots, q_{n}\right\rangle\right)$. For each variable $x_{i}$ in the domain of $M$, we define the set of variables $V\left(x_{i}\right)=\left\{x_{\left\langle q_{1}, \ldots, q_{n}\right\rangle^{j}}^{i} \mid q_{i} \in F_{i} \wedge\left\langle q_{1}, \ldots, q_{n}\right\rangle^{j} \in Q\right\}$. We define the domain $V$ of the mapping $C$ as $\bigcup_{i \in\{1, \ldots, n\}}\left(V\left(x_{i}\right)\right)$, and the image of each $x_{q}^{i}$ by $C$ as $q$. Finally, let $\Theta$ be the set of substitutions $\varphi$ over $\left\{x_{1}, \ldots, x_{n}\right\}$ satisfying $\varphi\left(x_{i}\right) \in V\left(x_{i}\right)$. We compute $S^{\prime}$ as a minimal set satisfying that each one of its terms is structurally equal to some term in $\{\varphi(s) \mid s \in S \wedge \varphi \in \Theta\}$, and vice-versa (i.e. $S^{\prime}$ is computed from $\{\varphi(s) \mid s \in S \wedge \varphi \in \Theta\}$ by removing repetitions modulo structural equality). Moreover, we force the terms in $S^{\prime}$ to do not share variables, by renaming them in $S^{\prime}$, and defining them in $V$ and $C$ whenever it is necessary. Obviously, each term in $S^{\prime}$ is structurally similar to some term in $S$, and any two distinct terms in $S^{\prime}$ are not structurally equal. Each $V\left(x_{i}\right)$ has at most $|Q|$ variables. Thus, $\Theta$ has at most $|Q|^{|M|}$ substitutions, and hence $\left|S^{\prime}\right| \leq|S| \cdot|Q|^{|M|}$. Generating $S^{\prime}$ consists of considering all of such combinations of a term in $S$ and a substitution in $\Theta$. Thus, the time complexity for creating $S^{\prime}$ from $S$ and $A$ is proportional to its size, i.e., is in $\mathcal{O}\left(\|S\| \cdot|Q|^{|M|}\right)$. In total, $\left\langle S^{\prime}, R=\langle A, C\rangle\right\rangle$ is constructed in exponential time w.r.t. $\|S\|+\|M\|$. 
3.3. Adding height constraints. Let $\left\langle S_{2}, R_{1}\right\rangle$ by the set of terms with single regular constraints that was obtained from $\left\langle S_{1}, M_{1}\right\rangle$ according to Lemma 3.6. Our algorithm proceeds by considering a term $s$ in $S_{2}$, and analyzing the kind of instances which are in $\mathrm{L}\left(\left\langle\{s\}, R_{1}\right\rangle\right)$ but not in $\mathrm{L}\left(\left\langle S_{2}-\{s\}, R_{1}\right\rangle\right)$. Depending on this analysis, it either concludes non-regularity of $\mathrm{L}\left(\left\langle S_{2}, R_{1}\right\rangle\right)$, or deduces that the height of the substitutions for some variables of $s$ can be bounded by $|Q|+2 H$, where $H$ is the maximum height of the terms in $S_{1}$. To manage this height constraint, we extend the notion of single regular constraint as follows.

Definition 3.7. A restricted regular constraint (over $\Sigma$ ) is a tuple $R=$ $\langle A, V, C, W, h\rangle$, where $W \subseteq V$ are sets of variables, $A=\langle Q, \Sigma, \delta\rangle$ is a DTA, $C$ is a mapping $C: V \rightarrow Q$, and $h$ is a natural number. The size $\|R\|$ of $R$ is $|V|+\|A\|$. A solution of $R$ is a mapping $\varphi: V \rightarrow T_{\Sigma}$ such that for all $x \in V$ it holds $\varphi(x) \in \mathrm{L}(A, C(x))$, and moreover, if $x \in W$ then height $(\varphi(x)) \leq h$. For a finite set $S \subseteq T_{\Sigma}(V)$, the pair $\langle S, R\rangle$ is a set of terms with restricted regular constraints. The language $\mathrm{L}(\langle S, R\rangle)$ of $\langle S, R\rangle$ is $\{t \mid \exists \varphi, s:(t=\varphi(s) \wedge s \in S \wedge \varphi$ is a solution of $R)\}$. $A$ term in $\mathrm{L}(\langle S, R\rangle)$ is also called an instance of $\langle S, R\rangle$.

Obviously, the set of terms with single regular constraints $\left\langle S_{2}, R_{1}=\langle A, C\rangle\right\rangle$ can be transformed into the set of terms with restricted regular constraints $\left\langle S_{2}, R_{2}=\right.$ $\langle A, V, C, \emptyset,|Q|+2 H\rangle\rangle$, and the represented language is preserved, i.e. $\mathrm{L}\left(\left\langle S_{2}, R_{1}\right\rangle\right)=$ $\mathrm{L}\left(\left\langle S_{2}, R_{2}\right\rangle\right)$. For a restricted regular constraint $\langle S, R\rangle$, we can define the infiniteinstances property analogously to Definition 3.2. where it is defined for a set of terms with regular constraints. As mentioned before, when a term in $S$ satisfies the infiniteinstances property, then $\mathrm{L}(\langle S, M\rangle)$ is not regular [7]. Exactly the same thing, with the same proof, can be said about a set of terms with restricted regular constraints $\langle S, R\rangle$.

LEMma 3.8. Let $\langle S, R\rangle$ be a set of terms with restricted regular constraints. Let $s$ be a term satisfying the infinite-instances property in $\langle S, R\rangle$. Then, $\mathrm{L}(\langle S, R\rangle)$ is not regular.

In order to make the paper self-contained, we prove this result. The proof is simplified and adapted to the case of restricted regular constraints.

Proof. We prove the lemma by contradiction, i.e. we assume that there exists DTA $B=\left\langle Q_{B}, \Gamma, \delta_{B}, F_{B}\right\rangle$ recognizing $\mathrm{L}(\langle S, R\rangle)$ in order to reach a contradiction.

By the assumptions, there exists a variable $x$ with more than one occurrence in $s$, and infinite instances $\varphi_{1}(s), \varphi_{2}(s), \ldots$ of $\langle\{s\}, R\rangle$ which are not instances of $\langle S-\{s\}, R\rangle$, and satisfying $\varphi_{i}(x) \neq \varphi_{j}(x)$ for all $j>i \geq 1$.

Let $R$ be $\langle A, V, C, W, h\rangle$, let $A$ be $\left\langle Q_{A}, \Gamma, \delta_{A}\right\rangle$, and let $H$ be the maximum height of the terms in $S$. Let $p_{1}$ be one of the positions in $s$ where $x$ occurs.

Since the instances $\varphi_{i}(s)$ are not in $\langle S-\{s\}, R\rangle$ and are different on $x$, there is a solution $\varphi\left(\varphi=\varphi_{i}\right.$ for some $\left.i \geq 1\right)$ of $R$ satisfying that $\varphi(s)$ is not an instance of $\langle S-\{s\}, R\rangle$ and height $(\varphi(x))>H+h+\left|Q_{A}\right| \cdot\left|Q_{B}\right|$. Let $p_{2}$ be a position such that $p_{1} \cdot p_{2}$ is a position of $\varphi(s),\left|p_{1} \cdot p_{2}\right|=H+h$ and height $\left(\varphi(s) /\left(p_{1} \cdot p_{2}\right)\right)>\left|Q_{A}\right|$. $\left|Q_{B}\right|$. By a simple pumping argument, there exist positions $p_{3}$ and $p_{4}$ satisfying that $p_{1} \cdot p_{2} \cdot p_{3} \cdot p_{4}$ is a position of $\varphi(s),\left|p_{4}\right| \geq 1, A\left(\varphi(s) /\left(p_{1} \cdot p_{2} \cdot p_{3} \cdot p_{4}\right)\right)=A\left(\varphi(s) /\left(p_{1} \cdot p_{2} \cdot p_{3}\right)\right)$ and $B\left(\varphi(s) /\left(p_{1} \cdot p_{2} \cdot p_{3} \cdot p_{4}\right)\right)=B\left(\varphi(s) /\left(p_{1} \cdot p_{2} \cdot p_{3}\right)\right)$.

Let $\bar{H}$ be height $(\varphi(s))$. Let $D$ be the context $\left(\varphi(s) /\left(p_{1} \cdot p_{2} \cdot p_{3}\right)\right)\left[p_{4} \leftarrow \bullet\right.$. We consider the term $t=\varphi(s)\left[p_{3} \leftarrow D^{\bar{H}}\left[\varphi(s) / p_{4}\right]\right]$. Note that $t$ is accepted by $B$. Thus, in order to reach a contradiction, it suffices to see that $t$ is not an instance of $\langle S, R\rangle$. It is clearly not an instance of $\langle s, R\rangle$, since we have the term $\varphi(s)\left[p_{3} \leftarrow D^{\bar{H}}\left[\varphi(s) / p_{4}\right]\right] / p_{1}$ as a subterm in $t$ at a position of $x$ in $s$, and the term $\varphi(s) / p_{1}$ as a subterm in $t$ at 
another position of $x$ in $s$. Thus, it rests to see that $t$ is not an instance of $\left\langle\left\{s^{\prime}\right\}, R\right\rangle$ for each $s^{\prime}$ in $S-\{s\}$.

For each term $s^{\prime}$ in $S-\{s\}$, we know that the term $\varphi(s)$ is not an instance of $\left\langle\left\{s^{\prime}\right\}, R\right\rangle$, and this has to be due to one of the following reasons:

(a) There is a position $q$ in $\operatorname{Pos}_{\mathrm{nv}}\left(s^{\prime}\right)$ satisfying that $q$ is not in $\operatorname{Pos}(\varphi(s))$,

(b) There is a position $q$ in $\operatorname{Pos}(\varphi(s)) \cap \operatorname{Pos}_{\mathrm{nv}}\left(s^{\prime}\right)$ satisfying $\varphi(s)[q] \neq s^{\prime}[q]$,

(c) There is a position $q$ in $\operatorname{Pos}(\varphi(s)) \cap \operatorname{Pos}_{\mathrm{v}}\left(s^{\prime}\right)$ satisfying $A(\varphi(s) / q) \neq C\left(s^{\prime}[q]\right)$,

(d) There are positions $q$ and $q^{\prime}$ in $\operatorname{Pos}(\varphi(s)) \cap \operatorname{Pos}_{\mathrm{v}}\left(s^{\prime}\right)$ satisfying $s^{\prime}[q]=s^{\prime}\left[q^{\prime}\right]$ and $\left.\varphi(s)\right|_{q} \neq\left.\varphi(s)\right|_{q^{\prime}}$.

(e) There is a position $q$ in $\operatorname{Pos}(\varphi(s)) \cap \operatorname{Pos}_{\mathrm{v}}\left(s^{\prime}\right)$ satisfying $s^{\prime}[q] \in W$ and $\operatorname{height}(\varphi(s))>h$.

In cases (a), (b), (c) and (e) it is straightforward that $t$ is not an instance of $\left\langle\left\{s^{\prime}\right\}, R\right\rangle$ by the same reason. Thus, assume we are in case (d). If both $q$ and $q^{\prime}$ are disjoint with $p_{1} \cdot p_{2}$, then $t / q=\varphi(s) / q \neq \varphi(s) / q^{\prime}=t / q^{\prime}$, and hence, $t$ is not an instance of $\left\langle\left\{s^{\prime}\right\}, R\right\rangle$. If one of $q$ or $q^{\prime}$, say $q$, is a prefix of $p_{1} \cdot p_{2}$, then, $t / q \neq t / q^{\prime}$ also holds, because height $(t / q)>\bar{H} \geq \operatorname{height}\left(t / q^{\prime}\right)$. Therefore, $t$ is not an instance of $\left\langle\left\{s^{\prime}\right\}, R\right\rangle$ in any case, and this concludes the proof.

For the particular case of a singleton $S=\{s\}$, Lemma 3.8 implies the following statement.

COROLlaRY 3.9. Let $\langle\{s\}, R\rangle$ be a set of terms with restricted regular constraints. Then, $\mathrm{L}(\langle\{s\}, R\rangle)$ is regular if and only if for each variable $x$ occurring at least twice in $s$, either $|\mathrm{L}(A, C(x))| \neq \infty$ or $x \in W$.

The previous corollary naturally leads to the following definition of regular term.

Definition 3.10. Let $R=\langle A, V, C, W, h\rangle$ be a restricted regular constraint. $A$ term $s \in T_{\Sigma}(V)$ is regular with respect to $R$ if for each variable $x$ occurring at least twice in $s$, either $|\mathrm{L}(A, C(x))| \neq \infty$ or $x \in W$.

3.4. Determining a term. At this point, we want to test whether a term $s$ satisfies the infinite-instances property with respect to $S_{2}$, that is, we want to analyze the instances of $\left\langle\{s\}, R_{2}\right\rangle$ which are not instances of $\left\langle S_{2}-\{s\}, R_{2}\right\rangle$. To make this problem easier, it would be good to have $s$ determined at all non-variable positions of the terms in $S_{2}$, according to the following definition.

DeFINITION 3.11. For a position $p$ and a term $s \in T_{\Sigma}(V)$, we say that $s$ is determined at $p$ if either $p \in \operatorname{Pos}_{\mathrm{nv}}(s)$ or there is a prefix $p^{\prime}$ of $p$ such that $s\left[p^{\prime}\right]$ is a constant symbol, i.e., it is in $\Sigma^{(0)}$. The term $s$ is determined at a set of positions $P$ if it is determined at each $p \in P$.

One of the nice (and obvious) properties of determined positions $p$ of $s$ is that, for any substitution $\varphi$ mapping variables to terms, the symbol $\varphi(s)[p]$ is either undefined or coincides with $s[p]$.

Lemma 3.12. Let $p$ be a position and $s$ a term determined at $p$. Let $\varphi_{1}, \varphi_{2}$ be mappings from variables to $T_{\Sigma}$. Either $p$ is not a position of both $\varphi_{1}(s)$ and $\varphi_{2}(s)$, or $\varphi_{1}(s)[p]=\varphi_{2}(s)[p]$.

Proof. No prefix $p^{\prime}$ of $p$ is such that $s\left[p^{\prime}\right]$ is a variable. Hence, for every substitution $\varphi$, we have that $\varphi(s)$ is undefined at $p$ if so was $s$, or that $\varphi(s)[p]=s[p]$ if not.

Another nice property of determined positions is that, given a term $s$, a restricted regular constraint $R$, and a set of positions $P$, then, a set of terms $s_{1}, \ldots, s_{k}$, all of them determined at $P$, can be generated in exponential time on $|\operatorname{Prefixes}(P)|$, such that $\{s\}$ and $\left\{s_{1}, \ldots, s_{k}\right\}$ represent the same language. The idea of determining a term at a set of positions was already used in 7 .

Lemma 3.13. Let $R=\langle A, V, C, W, h\rangle$ be a restricted regular constraint, where 
$A=\langle Q, \Sigma, \delta\rangle$. Let $s$ be a term in $T_{\Sigma}(V-W)$ and let $P$ be a set of positions. It can be computed in time $\mathcal{O}\left(|s| \cdot|\operatorname{Prefixes}(P)| \cdot\|R\|^{|\operatorname{Prefixes}(P)|}\right)$ an extension $R^{\prime}=$ $\left\langle A, V^{\prime}, C^{\prime}, W, h\right\rangle$ of $R$ and a set of terms $\left\{s_{1}, \ldots, s_{k}\right\}$ in $T_{\Sigma}\left(V^{\prime}\right)$ satisfying the following properties.

- $s_{1}, \ldots, s_{k}$ are determined at $P$.

- $\mathrm{L}\left(\left\langle\left\{s_{1}, \ldots, s_{k}\right\}, R^{\prime}\right\rangle\right)=\mathrm{L}(\langle\{s\}, R\rangle)$.

- Each $s_{i}$ can be obtained from $s$ through a substitution which replaces each variable by a term with height bounded by the maximum length of a position in $P$.

- $k \leq|\delta|^{|\operatorname{Prefixes}(P)|}$.

- For each $i$ in $\{1, \ldots, k\},\left|s_{i}\right|$ is bounded by $3 \cdot|\operatorname{Prefixes}(P)| \cdot|s|$.

Proof. We start with $\left\langle S_{1}^{\prime}, R_{1}^{\prime}\right\rangle=\langle\{s\}, R\rangle$ and transform it iteratively, while preserving the represented language, into new pairs $\left\langle S_{2}^{\prime}, R_{2}^{\prime}\right\rangle, \ldots,\left\langle S_{f}^{\prime}, R_{f}^{\prime}\right\rangle$, where we denote $S_{f}^{\prime}=\left\{s_{1}, \ldots, s_{k}\right\}$ and $R_{f}^{\prime}=R^{\prime}$. Let $s^{\prime}$ be a term of $S_{i}^{\prime}$ which is not determined at some $p \in \operatorname{Prefixes}(P) \cap \operatorname{Pos}_{\mathrm{v}}\left(s^{\prime}\right)$, and let $R_{i}^{\prime}=\left\langle A, V_{i}, C_{i}, W, h\right\rangle$ be the $i$-th constraint. Let $y=s^{\prime}[p]$ and let $q=C_{i}(y)$. The DTA $A$ has a finite number of transitions of the form $g\left(q_{1}, \ldots, q_{m}\right) \rightarrow q$, where $q, q_{1}, \ldots, q_{m} \in Q$ and $g \in \Sigma^{(m)}$ for $m \leq 2$, by the assumption on $\Sigma$. For each such transition, we construct the substitution $\gamma_{g, q_{1}, \ldots, q_{m}, q}=\left[y \leftarrow g\left(z_{1}, \ldots, z_{m}\right)\right]$ where $z_{1}, \ldots, z_{m}$ are new variables. Let $V_{i+1}^{\prime}$ be the union of these new sets of variables for all such transitions. Let $C_{i+1}^{\prime}$ be the union of all sets $\left\{\left(z_{1}, q_{1}\right), \ldots,\left(z_{m}, q_{m}\right)\right\}$ for all such transitions. We set $V_{i+1}:=V_{i+1}^{\prime} \cup V_{i}$ and $C_{i+1}:=C_{i+1}^{\prime} \cup C_{i}$. Finally, we set $S_{i+1}^{\prime}:=\left(S_{i}^{\prime}-\left\{s^{\prime}\right\}\right) \cup S^{\prime \prime}$, where $S^{\prime \prime}$ is the set of terms obtained by applying all the substitutions $\gamma_{g, q_{1}, \ldots, q_{m}, q}$ to $s^{\prime}$. Clearly, $\mathrm{L}\left(\left\langle S_{i+1}^{\prime}, R_{i+1}^{\prime}\right\rangle\right)$ coincides with $\mathrm{L}\left(\left\langle S_{i}, R_{i}\right\rangle\right)$.

At each of the $|\operatorname{Prefixes}(P)|$ positions we apply at most $|\delta|$ different substitutions

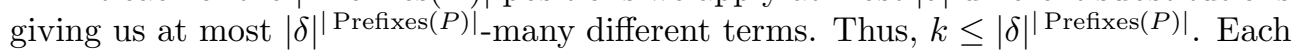
substitution $\gamma_{g, q_{1}, \ldots, q_{m}, q}$ increases the size of a term $s^{\prime}$ by the arity $m$ of $g$, which is at most 2 , and the variable replaced has at most $|s|$ occurrences in $s^{\prime}$. Thus, $|s|+|\operatorname{Prefixes}(P)|+2 \cdot|\operatorname{Prefixes}(P)| \cdot|s| \leq 3 \cdot|\operatorname{Prefixes}(P)| \cdot|s|$ bounds the size of each $s_{i}$.

Note that only those variables $y$ which appear at some position $p \in P$ may be replaced by some $\gamma_{g, q_{1}, \ldots, q_{m}, q}=\left[y \leftarrow g\left(z_{1}, \ldots, z_{m}\right)\right]$. Since the new variables $z_{i}$ always appear one position deeper than the variable $y$ they substitute, it follows that, in the process described, no variable of $s$ can be replaced by a term of height larger than the maximum length of the positions in $P$. $\square$

3.5. Structurally subsumed terms. Let $s$ be a term determined at all the non-variable positions of the terms in $S_{2}$. In order to check the infinite-instances property, our goal is to characterize the set $\mathrm{L}\left(\left\langle\{s\}, R_{2}\right\rangle\right)-\mathrm{L}\left(\left\langle S_{2}-\{s\}, R_{2}\right\rangle\right)$. Recall that $\operatorname{StructDiff}\left(S_{2}\right)$ is bounded by the initial $\left|S_{1}\right|$. This can be used to discard many terms in $S_{2}$ having no common instance with $\left\langle\{s\}, R_{2}\right\rangle$. To this end, we introduce the following notions. Let $A$ be a DTA and $C$ a mapping from variables to states of $A$. For a term $s \in T_{\Sigma}(V)$, we define $C(s):=A(s[x \leftarrow C(x) \mid x \in V])$. If $C$ is clear from the context, we denote a term $s$ by $s^{q}$ for $q=C(s)$, or as $f^{q}\left(s_{1}, \ldots, s_{m}\right)$ if $s=f\left(s_{1}, \ldots, s_{m}\right)$.

Definition 3.14. Let $R=\langle A, V, C, W, h\rangle$ be a restricted regular constraint over $\Sigma$, and let $s, t \in T_{\Sigma}(V)$. We say that $s$ is structurally subsumed by $t$ (with respect to $R$ ), if for all $p$ in $\operatorname{Pos}_{\mathrm{nv}}(t)$ it holds that $p$ is in $\operatorname{Pos}(s)$ and $t[p]=s[p]$, and moreover, for all $p$ in $\operatorname{Pos}(t)$ it holds that $C(t / p)=C(s / p)$.

Which terms in $\mathrm{L}\left(\left\langle S_{2}-\{s\}, R_{2}\right\rangle\right)$ can possibly have common instances with $s$ ? If 
$t$ structurally subsumes $s$, then they potentially have common instances (this depends on the equality constraints imposed by duplicated variables in $s$ and $t$ ). For instance, $t=f(x, x)$ structurally subsumes $s=f(a, b)$ if $C(x)=C(a)=C(b)$, but obviously $s$ and $t$ do not have common instances. What happens if $t$ does not structurally subsume $s$ ? Does this imply that $s$ and $t$ do not have common instances? Unfortunately not: $t=f(x, a)$ does not structurally subsume $s=f(a, y)$, but if $C(y)=C(x)$ and $a \in \mathrm{L}(A, C(x))$ then $\langle\{t\}, R\rangle$ and $\langle\{s\}, R\rangle$ share $f(a, a)$ as instance. At this point, the benefits of determining a term come into play.

LEMMA 3.15. Let $R=\langle A, V, C, W, h\rangle$ be a restricted regular constraint over $\Sigma$ and let $s, t \in T_{\Sigma}(V)$. If $s$ is determined at $\operatorname{Pos}_{\mathrm{nv}}(t)$ and $s$ is not structurally subsumed by $t$, then $\mathrm{L}(\langle\{s\}, R\rangle)$ and $\mathrm{L}(\langle\{t\}, R\rangle)$ are disjoint.

Proof. With the conditions of the lemma, and according to Definition 3.14, either it exists a position $p \in \operatorname{Pos}_{\mathrm{nv}}(t) \subseteq \operatorname{Pos}_{\mathrm{nv}}(s)$ such that $t[p] \neq s[p]$, or it exists a position $p \in \operatorname{Pos}(t) \subseteq \operatorname{Pos}(s)$ such that $C(t / p) \neq C(s / p)$. In the former case it is clear that all instances of $\langle\{s\}, R\rangle$ and $\langle\{t\}, R\rangle$ differ at $p$; in the latter case, the result follows from the fact that $\mathrm{L}(A, q)$ and $\mathrm{L}\left(A, q^{\prime}\right)$ are disjoint if $q \neq q^{\prime}$.

Moreover, when two terms are structurally similar but not structurally equal, they cannot both structurally subsume a third term.

LEMMA 3.16. Let $R=\langle A, V, C, W, h\rangle$ be a restricted regular constraint over $\Sigma$ and $s, t_{1}, t_{2} \in T_{\Sigma}(V)$. Assume that $t_{1}$ and $t_{2}$ are structurally similar but not structurally equal, and that $s$ is structurally subsumed by $t_{1}$. Then $s$ is not structurally subsumed by $t_{2}$.

Proof. If two terms $t_{1}$ and $t_{2}$ are structurally similar but not structurally equal, then there is a position $p \in \operatorname{Pos}_{\mathrm{v}}\left(t_{1}\right)=\operatorname{Pos}_{\mathrm{v}}\left(t_{2}\right)$ such that $C\left(t_{1}[p]\right) \neq C\left(t_{2}[p]\right)$. Now $t_{1}$ structurally subsumes $s$, so $C\left(t_{1}[p]\right)=C(s[p])$; this prevents $t_{2}$ from subsuming $s$.

$\square$

Recall that, by Lemma 3.6 , we can choose at most $\left|S_{1}\right|$ non-structurally similar terms in $S_{2}$. This fact, combined with Lemma 3.16, implies that at most $\left|S_{1}\right|-1$ terms in $S_{2}-\{s\}$ structurally subsume $s$. Since, by assumption, $s$ is determined at all non-variable positions of terms in $S_{2}$, then, by Lemma 3.15, only those $\left|S_{1}\right|-1$ terms may have common instances with $s$. Thus, when analyzing the instances of $\left\langle\{s\}, R_{2}\right\rangle$ which are not instances of $\left\langle S_{2}-\{s\}, R_{2}\right\rangle$, we can first choose the subset $S_{3}$ of terms in $S_{2}-\{s\}$ which structurally subsume $s$ (because they are the only possible ones to have common instances with $s$ ), and study which instances of $\left\langle\{s\}, R_{2}\right\rangle$ are not instances of $\left\langle S_{3}, R_{2}\right\rangle$. Note that $\left|S_{3}\right| \leq\left|S_{1}\right|-1$.

As mentioned before, if $t$ structurally subsumes $s$, then whether they have common instances or not, depends on the equality constraints imposed by duplicated variables. Since our restricted regular constraints also require that $\varphi(x) \leq h$ for $x \in W$, it means that $\varphi(s)$ can only be an instance of $t$ if the height of $\varphi(s) / p$ is smaller than or equal to $h$ whenever $t[p] \in W$.

LEMMA 3.17. Let $R=\langle A, V, C, W, h\rangle$ be a restricted regular constraint. Let $s$ and $t$ be terms such that $s$ is structurally subsumed by $t$ with respect to $R$, and $\operatorname{Vars}(s) \cap W=\emptyset$. Let $\varphi(s)$ be an instance of $\langle\{s\}, R\rangle$. Then $\varphi(s)$ is an instance of $\langle\{t\}, R\rangle$ if and only if

- for all $p, q$ in $\operatorname{Pos}_{\mathrm{v}}(t)$ such that $t[p]=t[q]$ it holds $\varphi(s) / p=\varphi(s) / q$, and

- for all $p$ in $\operatorname{Pos}_{\mathrm{v}}(t)$ such that $t[p] \in W$ it holds height $(\varphi(s) / p) \leq h$.

Proof. Since $s$ is structurally subsumed by $t$, an instance $\varphi^{\prime}(t)$ of $t$ coincides with $\varphi(s)$ if and only if $\varphi^{\prime}(t) / p=\varphi(s) / p$ for every $p$ in $\operatorname{Pos}_{\mathrm{V}}(t)$. But this condition uniquely determines $\varphi^{\prime}$, i.e. $\varphi(s)$ is an instance $\varphi^{\prime}(t)$ of $t$ if and only if $\varphi^{\prime}$ is defined as 
$\varphi^{\prime}(t[p]):=\varphi(s) / p$, for every $p$ in $\operatorname{Pos}_{\mathrm{v}}(t)$. This definition of $\varphi^{\prime}$ is correct (i.e. uniquely defined for each variable) if and only if for all $p, q$ in $\operatorname{Pos}_{\mathrm{v}}(t)$ such that $t[p]=t[q]$ it holds $\varphi(s) / p=\varphi(s) / q$. Thus, the first item is necessarily satisfied. Moreover, by the assumptions of the lemma, for all $p \in \operatorname{Pos}(t)$ it holds $C(t / p)=C(s / p)$. Thus, the instance $\varphi^{\prime}(t)$ of $t$ is also an instance of $\langle t, R\rangle$ if and only if for all $p \in \operatorname{Pos}_{\mathrm{v}}(t)$ such that $t[p] \in W$, it holds height $(\varphi(s) / p) \leq h$, as required by the second item of the lemma. $\mathrm{c}$

3.6. Formulas representing instances. By using Lemma 3.17 we are able to characterize the instances of $\left\langle\{s\}, R_{2}\right\rangle$ which are not instances of $\left\langle S_{3}, R_{2}\right\rangle$ as the solutions of a formula $F$ which is a disjunction of conjunctions with inequalities between terms and height restrictions of terms as predicates, and a single regular constraint for the variables.

Definition 3.18. Let $V$ be a finite set of variables. A formula with inequality and height predicates $F$ (over $V$ ) is a disjunction of conjunctions of predicates of the form $s \neq t$ and height $(s)>h$, where $s, t \in T_{\Sigma}(V)$ and $h$ is a natural number. A constrained formula of order $n$ is a triple $\langle F, A, C\rangle$, where $A=\langle Q, \Sigma, \delta\rangle$ is a 1-or- $(n+$ 1) DTA, $F$ is a formula with inequality and height predicates where every conjunction has at most $n$ predicates, and $C$ is a total function $C: V \rightarrow Q$. Moreover, for each predicate height $(s)>h$ we require that $h$ is greater than or equal to $|Q|+\operatorname{height}(s)$. A solution of $\langle F, A, C\rangle$ is a substitution $\varphi: V \rightarrow T_{\Sigma}$ such that $A(\varphi(x))=C(x)$ for each $x \in V$ and $\varphi(F)$ evaluates to true by interpreting $\neq$, height, and $>$ in the natural way. The set of all solutions is denoted $\operatorname{Sol}(\langle F, A, C\rangle)$.

We now construct a constrained formula for a given set of terms $S$ and term $s$. Denote by $\operatorname{selPos}(S)$ the set of functions $P: S \rightarrow \cup_{s^{\prime} \in S}\left(\operatorname{Pos}_{\mathrm{v}}\left(s^{\prime}\right)\right)$ such that for each $s^{\prime} \in S, P\left(s^{\prime}\right) \in \operatorname{Pos}_{\mathrm{v}}\left(s^{\prime}\right)$. Let $W$ be a set of variables. We define $\operatorname{selPos}(S, W)$ as the subset of functions $P$ of $\operatorname{selPos}(S)$ such that for each $s^{\prime} \in S, s^{\prime}\left[P\left(s^{\prime}\right)\right] \in W$.

Definition 3.19. Let $S$ be a set of terms, and let $R=\langle A, V, C, W, h\rangle$ be a restricted regular constraint, where $A=\langle Q, \Sigma, \delta\rangle$ is a 1-or- $(|S|+1)$ DTA. Finally, let $s \in T_{\Sigma}(V-W)$ be a term that is structurally subsumed by all terms in $S$ with respect to $R$. Also suppose that $h$ is greater than or equal to $|Q|+\operatorname{height}(s)$. We define $\mathcal{F}(s, S, W, h)$ as

$$
\bigvee_{\alpha}\left(\bigwedge_{t \in S^{\prime}} s / P(t) \neq s / U(t) \bigwedge_{t \in S-S^{\prime}} \operatorname{height}(s / T(t))>h\right)
$$

where $\alpha$ says that $S^{\prime} \subseteq S ; P, U \in \operatorname{selPos}\left(S^{\prime}\right)$ such that for every $s^{\prime} \in S^{\prime}: P\left(s^{\prime}\right) \neq U\left(s^{\prime}\right)$ and $s^{\prime}\left[P\left(s^{\prime}\right)\right]=s^{\prime}\left[U\left(s^{\prime}\right)\right] ;$ and $T \in \operatorname{selPos}\left(S-S^{\prime}, W\right)$. Note that $\mathcal{F}(s, S, W, h)$ is a formula with inequality and height predicates and that $\langle\mathcal{F}(s, S, W, h), A, C\rangle$ is a constrained formula of order $|S|$.

According to Lemma 3.17 the instances of $\langle\{s\}, R\rangle$ that are not instances of $\langle S, R\rangle$ are precisely the terms $\varphi(s)$ with $\varphi \in \operatorname{Sol}(\langle\mathcal{F}(s, S, W, h), A, C\rangle)$. We state this in the following lemma.

Lemma 3.20. Let $R=\langle A, V, C, W, h\rangle$ be a restricted regular constraint, where $A$ is $\langle Q, \Sigma, \delta\rangle$. Let $S$ be a set of terms and let $s \in T_{\Sigma}(V-W)$ be a term that is structurally subsumed by all terms in $S$, and such that $h \geq|Q|+\operatorname{height}(s)$. Then, $\mathrm{L}(\langle\{s\}, R\rangle)-\mathrm{L}(\langle S, R\rangle)=\operatorname{Sol}(\langle\mathcal{F}(s, S, W, h), A, C\rangle)$.

Our goal is to decide whether $\left\langle\{s\}, R_{2}\right\rangle$ has infinitely many instances, all of them different on a certain variable $x$, and all of them not instances of $\left\langle S_{3}, R_{2}\right\rangle$. We do not solve this problem for an arbitrary restricted regular constraint $R$. Recall that 
$\left|S_{3}\right| \leq\left|S_{1}\right|-1, R_{2}$ is of the form $\left.\langle A, V, C, W,|Q|+2 H)\right\rangle$ where $H$ is the maximum height of a term in $S_{1}$, height $(s) \leq 2 H$ and $A$ is a 1-or- $\left|S_{1}\right|$ DTA. Our problem now translates to the constrained formula $\left\langle\mathcal{F}\left(s, S_{3}, W,|Q|+2 H\right), A, C\right\rangle$, which is of order $\left|S_{3}\right|$ due to the particularities of $R_{2}$; i.e., we need to decide whether $\left\langle\mathcal{F}\left(s, S_{3}, W, h\right), A, C\right\rangle$ has infinite solutions and all of them different on a concrete variable $x$. To this end we proceed by transforming this formula by means of the set of rules described in Figure 3.1. The following lemma states that the inference system preserves the set of solutions.

Remove-insat1: $\quad \frac{C \vee(t \neq t \wedge D)}{C}$

Remove-insat2: $\quad \frac{C \vee\left(s^{q} \neq t^{q} \wedge D\right)}{C}$

where $|\mathrm{L}(A, q)|=1$.

Remove-sat1: $\quad \frac{C \vee\left(s^{q} \neq t^{q^{\prime}} \wedge D\right)}{C \vee(D)}$

where either $q \neq q^{\prime}$, or $s[\varepsilon], t[\varepsilon]$ are not variables and $s[\varepsilon] \neq t[\varepsilon]$.

Remove-sat2: $\quad \frac{C \vee\left(x^{q} \neq t^{q} \wedge D\right)}{C \vee(D)}$

where $t$ is not $x$ and $x \in \operatorname{Vars}(t)$.

Decompose: $\quad \frac{C \vee\left(f^{q}\left(s_{1}, \ldots, s_{m}\right) \neq f^{q}\left(t_{1}, \ldots, t_{m}\right) \wedge D\right)}{C \vee \bigvee_{i \in\{1, \ldots m\}}\left(s_{i} \neq t_{i} \wedge D\right)}$

Decrease-height: $\frac{C \vee\left(\operatorname{height}\left(f^{q}\left(s_{1}, \ldots, s_{m}\right)\right)>h \wedge D\right)}{C \vee \bigvee_{i \in\{1, \ldots m\}}\left(\operatorname{height}\left(s_{i}\right)>h-1 \wedge D\right)}$

where $\mathrm{L}(A, q)$ is infinite, and $h>|Q|$.

Remove-height: $\quad \frac{C \vee\left(\text { height }\left(s^{q}\right)>h \wedge D\right)}{C}$

where $\mathrm{L}(A, q)$ is finite, and $h \geq|Q|$.

FIG. 3.1. Inference rules for transforming formulas into final formulas.

Lemma 3.21. If $\langle F, A, C\rangle$ is a constrained formula of order $n$ and $\langle F, A, C\rangle$ derives into $\langle G, A, C\rangle$ by the application of one inference rule of Figure [3.1, then $\langle G, A, C\rangle$ is a constrained formula of order $n$, and $\operatorname{Sol}(\langle G, A, C\rangle)=\operatorname{Sol}(\langle F, A, C\rangle)$.

Proof. It is clear that $\langle G, A, C\rangle$ is also a constrained formula of the same order than $\langle F, A, C\rangle$ : the DTA does not change; the number of predicates in a conjunction is never increased; and the only rule that adds a new height predicate, i.e., rule Decrease-height, reduces both by one the height of the left side term and the right side bound. 
To see that the solutions are preserved under the applications of the inference rules, we only need to observe that rules Remove-insat1, Remove-insat2, and Removeheight simply remove conjunctions that are impossible to satisfy; that rules Removesat1 and Remove-sat2 remove inequality statements from inside a conjunction which are always satisfied; and that rules Decompose and Decrease-height decompose a statement into an equivalent disjunction of statements. $\square$

Definition 3.22. A constrained formula $\langle F, A, C\rangle$ is final if no rule can be applied on $\langle F, A, C\rangle$.

The following lemma characterizes final formulas.

Lemma 3.23. Let $\langle F, A, C\rangle$ be a constrained formula of order $n$. Then, $\langle F, A, C\rangle$ is a final formula of order $n$ if and only if $F$ is a disjunction of conjunctions of the form

$$
\left(x_{1} \neq t_{1} \wedge \ldots \wedge x_{m} \neq t_{m} \wedge \operatorname{height}\left(y_{1}\right)>h_{1} \wedge \ldots \wedge \operatorname{height}\left(y_{k}\right)>h_{k}\right)
$$

where $k+m \leq n$, every $x_{i}$ is a variable not occurring in the corresponding $t_{i}$, every $C\left(x_{i}\right)$ coincides with its corresponding $C\left(t_{i}\right)$, every $\left|\mathrm{L}\left(A, C\left(x_{i}\right)\right)\right|>n$, and every $y_{i}$ is a variable satisfying $\left|\mathrm{L}\left(A, C\left(y_{i}\right)\right)\right|=\infty$.

Proof. The right-to-left implication trivially follows by inspecting that no inference rule can be applied on $F$. For the left-to-right implication, assume that $\langle F, A, C\rangle$ is a final formula. First, let $s \neq t$ be any inequality predicate of $F$. The terms $s$ and $t$ are different since rule Remove-insat1 is not applicable. One of both has to be a variable: otherwise, one of Remove-sat1 or Decompose is applicable. Without loss of generality, let $s$ be a variable $x$. Then, $x$ cannot occur in $t$ : otherwise, rule Removesat2 is applicable (recall that $x=s$ and $t$ are different). The states $C(x)$ and $C(t)$ coincide: otherwise, rule Remove-sat1 is applicable. Moreover, $|\mathrm{L}(A, C(x))|>n$ : since $A$ is a 1 -or- $(n+1)$ DTA, $|\mathrm{L}(A, C(s))|$ is either 1 or greater than $n$, but it cannot be 1 because, otherwise, rule Remove-insat2 would be applicable. Second, let height $(u)>|Q|+h$ be any height predicate of $F$. The cardinality of $\mathrm{L}(A, C(u))$ must be infinite: otherwise rule Remove-height is applicable. Moreover, the term $u$ must be a variable: otherwise, rule Decrease-height is applicable.

The following lemma proves that any non-empty final formula of order $n$ has a solution, and moreover, if some variable $x$ has an infinite language, then there are infinitely many solutions all of them different on $x$.

Lemma 3.24. Let $V$ be a set of variables. Let $\langle F, A, C\rangle$ be any non-empty final formula (over $V$ ) of order $n$. Then, $\langle F, A, C\rangle$ has a solution. Moreover, if $x \in V$ satisfies that $|\{\mathrm{L}(A, C(x))\}|=\infty$, then there exists infinitely many solutions $\varphi_{1}, \varphi_{2}, \ldots$ of $\langle F, A, C\rangle$ such that all $\varphi_{1}(x), \varphi_{2}(x), \ldots$ are pairwise different.

Proof. Note that $F$ is a disjunction of conjunctions $\bigvee G_{i}$, where each $\left\langle G_{i}, A, C\right\rangle$ is also a non-empty final formula, and $\operatorname{Sol}\left(\left\langle G_{i}, A, C\right\rangle\right) \subseteq \operatorname{Sol}(\langle F, A, C\rangle)$ holds. Thus, we assume the simple case where $F=G_{1}$ is a single conjunction of predicates $\left(x_{1} \neq\right.$ $t_{1} \wedge \ldots \wedge x_{m} \neq t_{m} \wedge \operatorname{height}\left(y_{1}\right)>h_{1} \wedge \ldots \wedge$ height $\left.\left(y_{k}\right)>h_{k}\right)$.

We construct a solution $\varphi$ of $\langle F, A, C\rangle$ by first defining $\varphi(x)=t$ for each variable $x$ satisfying that $\mathrm{L}(A, C(x))$ is a singleton language $\{t\}$ (note that this is the only possible election for $\varphi(x)$ in a solution). Then, we replace all occurrences of $x$ by $t$ in $F$. By Lemma 3.23, each occurrence of $x$ must be at a child position of some node in a $t_{i}$. After that, the resulting $F$ satisfies that no variable $x$ with $|\mathrm{L}(A, C(x))|=1$ occurs in $F$, but the left-hand sides of inequalities are still variables, and each $x_{i} \neq t_{i}$ satisfies that $x_{i}$ does not occur in $t_{i}$.

Now, we complete the definition of $\varphi$ by applying the process explained below. 
This process chooses a particular variable $x$ at each step, chooses a particular substitution $\varphi(x)$ for it, and then replaces all occurrences of $x$ in $F$ by $\varphi(x)$. Thus, the process terminates. Since $F$ is modified along the execution, it can lose the property of being a final formula: for example, when an equation $x=t$ occurs and $x$ is instantiated, it is no longer true that each equation has a variable in one of its sides. The election of each $\varphi(x)$ for each corresponding $x$ is done in a way such that, whenever a predicate is made variable-free, then it is trivially true.

(a) If all inequalities of $F$ with variables contain at least two distinct variables, then choose any variable $x$ occurring in them. Choose any variable-free term $t$ in $\mathrm{L}(A, C(x))$, also satisfying height $(t)>h$ if a predicate height $(x)>h$ occurs in $F$ (note that such a $t$ exists since, by Lemma 3.23, the language $\mathrm{L}(A, C(x))$ is infinite). Then, define $\varphi(x):=t$. Replace each occurrence of $x$ by $t$ in $F$. Jump to (a).

(b) If $F$ still contains a variable in some inequality, then choose an inequality $s_{i} \neq t_{i}$ with occurrences of just one variable $x$, i.e. satisfying $\operatorname{Vars}\left(s_{i}\right) \cup$ $\operatorname{Vars}\left(t_{i}\right)=\{x\}$. Without loss of generality, let $s_{1} \neq t_{1}, \cdots, s_{m^{\prime}} \neq t_{m^{\prime}}$ be the inequalities containing $x$ and no other variable than $x$. Choose a variable-free term $t$ in $\mathrm{L}(A, C(x))$, such that $\{x \mapsto t\}\left(s_{1} \neq t_{1} \wedge \cdots \wedge s_{m^{\prime}} \neq t_{m^{\prime}}\right)$ is true (note that this is possible since $|\mathrm{L}(A, C(x))|>n \geq m \geq m^{\prime}$, where $n$ is the order of the final formula $\langle F, A, C\rangle)$, and also satisfying height $(t)>h$ if a predicate height $(x)>h$ occurs in $F$ (as before, such a $t$ exists since in this case $\mathrm{L}(A, C(x))$ is infinite). Replace each occurrence of $x$ by $t$. Jump to (a).

(c) For each variable $x$ for which $\varphi$ is still not defined, choose any term $t$ in $\mathrm{L}(A, C(x))$ also satisfying height $(t)>h$ if a predicate height $(x)>h$ occurs in $F$, and define $\varphi(x):=t$. Replace each occurrence of $x$ by $t$.

For the case of variables $x$ with infinite $\mathrm{L}(A, C(x))$, when the process above chooses a value for them, it has an infinite number of possibilities. Hence, infinitely many solutions $\varphi$ can be found, all of them distinct on $\varphi(x)$.

Lemma 3.25. Let $s \in T_{\Sigma}(V-W)$ be a term determined at $\operatorname{Pos}_{\mathrm{nv}}(S)$, where $\langle S, R\rangle$ is a set of terms with restricted regular constraints. Let $R$ be of the form $\langle A, V, C, W, h\rangle$, where $A$ is a 1-or- $(|S|+1) D T A$ and $h \geq|Q|+\operatorname{height}(s)$. It is decidable in time $\mathcal{O}\left(2^{|S|} \cdot|s|^{2|S|+1}|S|\right)$ whether $\langle\{s\}, R\rangle$ has an instance not in $\mathrm{L}(\langle S, R\rangle)$. In the affirmative case, if a variable $x$ occurs at least twice in s and it satisfies $|\mathrm{L}(A, C(x))|=$ $\infty$, then s has infinitely many instances not in $\mathrm{L}(\langle S, R\rangle)$, and all of them different on $x$.

Proof. Let $F$ be $\mathcal{F}(s, S, W, h)$. The constrained formula $\langle F, A, C\rangle$ of order $|S|$ can be easily constructed in time $T=2^{|S|+1} \cdot|S| \cdot|s|^{2|S|+1}$, since it has no more than $2^{|S|} \cdot|s|^{2|S|}$ conjunctions, each of them with at most $|S|$ statements of size bounded by $2 \cdot|s|$. In fact, $2^{|S|} \cdot|s|^{2|S|}$ is also a bound for the total number of different conjunctions that may appear along the inference process. Thus, if we treat each conjunction once, by removing the generated ones that have been already treated, at most $2^{|S|} \cdot|s|^{2|S|}$ inference steps are executed. Each inference step takes time proportional to the size of a conjunction, which is bounded by $|S| \cdot 2 \cdot|s|$, multiplied by the maximum arity, which is 2 by our simplifying assumption. Thus, the total cost is $\mathcal{O}\left(2^{|S|} \cdot|s|^{2|S|+1}|S|\right)$.

$\square$

3.7. The algorithm. We summarize in Figure 3.2 the EXPTIME algorithm for deciding regularity of a set of terms with regular constraints.

The algorithm starts by transforming the input instance $\left\langle S_{1}, M_{1}\right\rangle$ into an equivalent set of terms with single regular constraints in exponential time, according to 


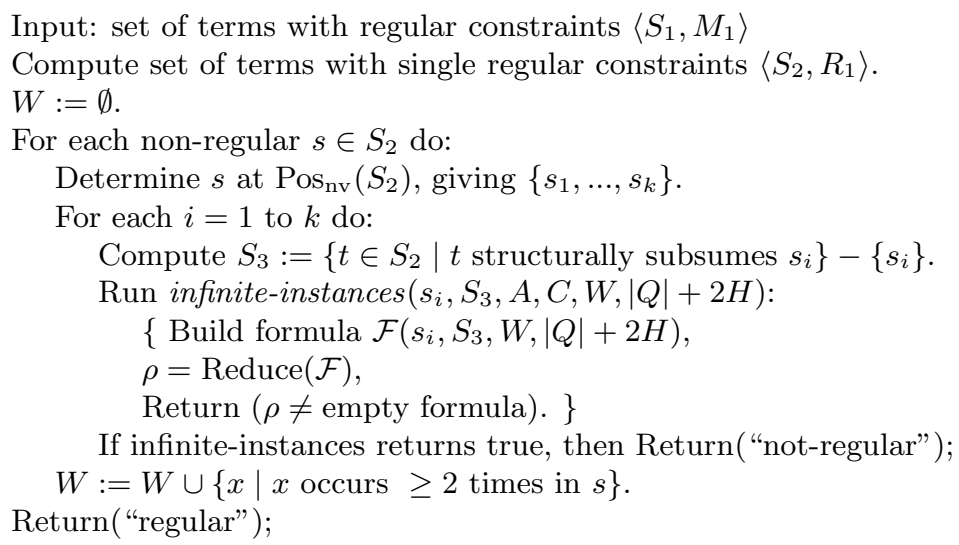

FIG. 3.2. The EXPTIME algorithm for deciding regularity.

Lemma 3.6. Then, the algorithm (implicitly) considers a restricted regular constraint $R_{2}=\langle A, V, C, W,|Q|+2 H\rangle$, where $W=\emptyset$ at the beginning. The determination of $s$ into $s_{1}, \ldots, s_{k}$ is done according to Lemma 3.13. This determination also takes exponential time on the size of the input instance $\left\langle S_{1}, M_{1}\right\rangle$, since Prefixes $\left(\operatorname{Pos}_{\mathrm{nv}}\left(S_{2}\right)\right)$ coincides with Prefixes $\left(\operatorname{Pos}_{\mathrm{nv}}\left(S_{1}\right)\right)$. Finally, the infinite-instances property can be also determined in exponential time, due to Lemma 3.25 and the fact that $\left|S_{3}\right| \leq\left|S_{1}\right|-1$. Thus, it follows from the previous lemmas that the algorithm runs in exponential time with respect to $\left\|S_{1}\right\|+\left|M_{1}\right|$.

Now we discuss the correctness of the algorithm. Let $R_{2}^{\prime}$ be the extension of $R_{2}$ obtained when determining $s$ into $s_{1}, \ldots, s_{k}$, according to Lemma 3.13. Assume the case where none of the $s_{i}$ satisfies the infinite instances property in $S_{2}$, and consider a concrete term $s_{i}$ satisfying that $\left\langle\left\{s_{i}\right\}, R_{2}\right\rangle$ has instances not in $\mathrm{L}\left(\left\langle S_{2}-\right.\right.$ $\left.\left.\{s\}, R_{2}\right\rangle\right)$. By Lemma 3.25 $s_{i}$ cannot have duplicated variables with associated infinite language. Now, consider a duplicated variable $x$ with infinite language and occurring at a position $p$ in $s$. By Lemma 3.13, $s_{i} / p$ has height bounded by $H$, and occurs at another position in $s_{i}$. Thus, all the variables $y$ occurring in $s_{i} / p$ are duplicated in $s_{i}$, and hence, $\mathrm{L}\left(\left\langle\{y\}, R_{2}^{\prime}\right\rangle\right)$ is finite. In particular, they can be instantiated by a term with height bounded by $|Q|$ in order to get an instance. Therefore, $\mathrm{L}\left(\left\langle\left\{s_{i} / p\right\}, R_{2}^{\prime}\right\rangle\right)$ is finite, and any instance $t$ of $\mathrm{L}\left(\left\langle\left\{s_{i}\right\}, R_{2}^{\prime}\right\rangle\right)$ satisfies height $(t / p) \leq|Q|+H$.

From the above considerations we conclude that all instances $t$ of $\left\langle\{s\}, R_{2}\right\rangle$ not in $\mathrm{L}\left\langle S-\{s\}, R_{2}\right\rangle$ satisfy the following statement: for each position $p$ with a duplicated variable in $s$, height $(t / p) \leq|Q|+H \leq|Q|+2 H$. Thus, by adding the duplicated variables in $s$ to $W$ we preserve the represented language.

TheOREM 3.26. The above algorithm solves RITRC in exponential time.

4. Concluding Remarks. In this contribution we have shown that the RITRC problem is EXPTIME-hard, and have presented a new algorithm that solves RITRC in exponential time. This problem is a particular case of the HOM problem [5]: given a DTA $A$ and a tree homomorphism $H$, is $H(\mathrm{~L}(A))$ regular? The decidability of this problem is a long-standing open question. The main problem is how to handle nonlinearity of $H$, and to determine in which cases it forces non-regularity of $H(\mathrm{~L}(A))$. Our algorithm gives some intuition about when non-linearity poses a real problem for the regularity of the represented set (it also gives an exponential time solution for the HOM problem in the case that non-linear rules are only applied at bounded depth of 
the input tree, cf. [7). But, it is still far from solving the general problem.

\section{REFERENCES}

[1] V. Benzaken, G. Castagna, H. Hosoya, B.C. Pierce, and S. Vansummeren, The Encyclopedia of Database Systems, Springer, 2009, ch. "XML Typechecking".

[2] H. Comon, M. Dauchet, R. Gilleron, C. Löding, F. Jacquemard, D. Lugiez, S. TiSON, AND M. TOMmASI, Tree automata techniques and applications. Available at http://www.grappa.univ-lille3.fr/tata, 2007.

[3] H. Comon And C. Delor, Equational formulae with membership constraints, Infor. and Comput., 112 (1994), pp. 167-216.

[4] J. Doner, Tree acceptors and some of their applications, J. Comp. Syst. Sci., 4 (1970), pp. 406451.

[5] Z. FüLöP, Undecidable properties of deterministic top-down tree transducers, Theoret. Comput. Sci., 134 (1994), pp. 311-328.

[6] F. Gécseg and M. Steinby, Tree languages, in Handbook of Formal Languages, Volume 3, G. Rozenberg and A. Salomaa, eds., Springer, 1997, ch. 1.

[7] G. Godoy, S. Maneth, And S. Tison, Classes of tree homomorphisms with decidable preservation of regularity, in FoSSaCS, vol. 4962 of LNCS, Springer, 2008, pp. 127-141.

[8] H. Hosoya And B. C. Pierce, Regular expression pattern matching for XML, J. Funct. Program., 13 (2003), pp. 961-1004.

[9] H. Hosoya, J. Vouillon, And B. C. Pierce, Regular expression types for XML, ACM Trans. Program. Lang. Syst., 27 (2005), pp. 46-90.

[10] G. Kucherov and M. Rusinowitch, Patterns in words versus patterns in trees: A brief survey and new results, in Ershov Memorial Conference, Springer, 1999, pp. 283-296.

[11] J.-L. LASSEZ AND K. MARRIOTT, Explicit representation of terms defined by counter examples, J. Automat. Reason., 3 (1987), pp. 301-317.

[12] J. Mezei and J. B. Wright, Algebraic automata and context-free sets, Inform. and Control, 11 (1967), pp. 3-29.

[13] M. Murata, D. Lee, M. Mani, and K. Kawaguchi, Taxonomy of XML schema languages using formal language theory., ACM Trans. Internet Techn., 5 (2005), pp. 660-704. 